\title{
Funcionalidad familiar, soporte de amigos y rendimiento académico en estudiantes de odontología
}

\author{
Family functionality, friend support and academic performance in Odontology college students \\ Guillermo Tamayo-Cabeza ${ }^{*}$ orcid.org/0000-0002-2252-701X \\ Arleth Hernández-Alvarez ${ }^{2}$ orcid.org/0000-0002-6920-2386 \\ Shyrley Díaz-Cárdenas ${ }^{2,3}$ orcid.org/0000-0003-1967-8981
}

1. Facultad de Medicina, Universidad de los Andes. Bogotá, Colombia.

2. Facultad de Odontología, Universidad de Cartagena. Cartagena, Colombia

3. Facultad de Odontología, Departamento Odontología Preventiva y Social. Universidad de Cartagena. Cartagena, Colombia

Fecha de recepción: Febrero 20 - 2021

Fecha de revisión: Junio 08 - 2021

Fecha de aceptación: Diciembre 20 - 2021

Tamayo-Cabeza G, Hernández-Torres A, Díaz-Cárdenas $S$. Funcionalidad familiar, soporte de amigos y rendimiento académico en estudiantes de odontología. Univ. Salud. 2022;24(1):18-28. DOI: https://doi.org/10.22267/rus.222401.263

\section{Resumen}

Introducción: Los factores relacionados con la funcionalidad familiar en estudiantes universitarios podrían asociarse con la deserción académica e impactar de manera negativa sobre el rendimiento académico. Objetivo: Describir las relaciones entre las características sociodemográficas y familiares, con la funcionalidad familiar, el soporte de amigos y el rendimiento académico en los estudiantes de odontología de una universidad pública de la ciudad de Cartagena. Materiales y métodos: Estudio de corte transversal, con una muestra de 438 estudiantes. Se utilizó un instrumento tipo encuesta sobre variables sociodemográficas, características familiares y de la vivienda. Los datos fueron analizados a partir de estadística descriptiva y análisis bivariados con la prueba Chi cuadrado de Pearson. Resultados: Se observó una relación estadísticamente significativa entre la edad y la funcionalidad familiar $(p=0,0029)$. Asimismo, se obtuvo una relación significativa entre la procedencia y el soporte de amigos $(p=0,0230)$, así como entre la baja escolaridad y la funcionalidad familiar $(p=0,0018)$. Conclusiones: La identificación de los factores que se relacionan con la funcionalidad familiar y el soporte de amigos pueden contribuir a determinar qué aspectos deben considerarse para el diseño e implementación de intervenciones académicas dirigidas a estudiantes del programa.

Palabras clave: Familia; amigos; rendimiento académico; estudiantes; odontología; salud familiar. (Fuente: DeCS, Bireme).

\begin{abstract}
Introduction: Academic dropout in college students could be associated with family functionality, which could also negatively impact their academic performance. Objective: To describe the relationship between sociodemographic and family characteristics with family functionality, friend support, and academic performance in Odontology students from a public university in the city of Cartagena (Colombia). Materials and methods: A cross-sectional study was conducted with 438 students. A survey-type instrument was used to obtain information regarding sociodemographic variables as well as family and home characteristics. Data were analyzed using descriptive statistics and bivariate analysis with a Pearson's Chi square test. Results: A statistically significant relationship was observed between students' age and family functionality $(p=0.0029)$. Likewise, a significant relationship was found between the origin of the students and friend support $(\mathrm{p}=0.0230)$, as well as between low education level and family functionality $(p=0.0018)$. Conclusions: The identification of factors related to family functionality and friend support can contribute to determining important aspects to be considered for the design and implementation of academic interventions focused on Odontology students.
\end{abstract}

Keywords: Family; friends; academic performance; students; dentistry; family health. (Source: DeCS, Bireme).

\footnotetext{
*Autor de correspondencia

Guillermo Tamayo-Cabeza

e-mail: gtamayocabeza@outlook.com
} 


\section{Introducción}

Muchos estudiantes cuando ingresan a la universidad, se encuentran en la mayoría de edad, que para Colombia es de 18 años. En esta etapa de la vida, se espera que el estudiante haya adquirido una madurez intelectual y física suficiente como para tener una voluntad válida para obrar algunos actos con autonomía e independencia; principalmente, a la hora de tomar decisiones, realizar gestiones al interior de la institución y responder académicamente por sus $\operatorname{compromisos}^{(1)}$.

Esta situación genera un desconocimiento sobre el entorno familiar que rodea al estudiante por parte de las instituciones académicas, lo cual constituye uno de los factores relacionados con su rendimiento académico(2). En ese entorno suceden dinámicas familiares como la forma de crianza de los hijos, ausencia de los padres por motivos laborales, consumo de alcohol y drogas, el tener hijos preferidos o no deseados, así como la separación de los padres. Estos son elementos que pueden generar deserción académica, ya que son características de disfuncionalidad familiar, la cual impacta de manera negativa sobre el rendimiento académico. El modelo de familia y la presencia de funcionalidad familiar pueden ser determinantes en la calidad del desempeño del estudiante y contribuir a que este alcance sus objetivos académicos(3,4).

Otros factores sociodemográficos y sociales pueden también conducir a la deserción académica, tales como la baja escolaridad de los padres, un bajo estrato socioeconómico, el hacinamiento y la falta de interés en las actividades académicas de los hijos(5). Así mismo, son relevantes las relaciones que se dan entre los amigos, lo cual corresponde a una fuente permanente de refuerzo a la identidad de los individuos y un soporte afectivo necesario para el logro de los objetivos académicos. Tanto la resiliencia como el apoyo familiar y de los amigos se constituyen como factores de afrontamiento que pueden disminuir las cargas de estrés académico en estudiantes universitarios ${ }^{(6,7)}$.

Dependiendo de la naturaleza de las carreras profesionales se podría generar mucho más estrés, como es el caso de carreras relacionadas con el área de la salud, al dedicar su quehacer profesional basado en la atención clínica de seres humanos(4). Un estudio demuestra que la odontología genera más estrés que la carrera de medicina(8); puesto que implica gran dedicación de horas de estudios, altos grados de estrés por exigencia académica, mayor permanencia en las instituciones académicas por la recarga de trabajos, atención de pacientes y subsidio de tratamientos bucales, altos costos de materiales odontológicos y muy poco tiempo para el descanso y el sueño( ${ }^{(9)}$. Además, algunos estudios dan cuenta de factores que influyen en el rendimiento académico en estudiantes de odontología, tales como: encontrarse en medio de la adolescencia(10,11), vivir en ambientes con disfuncionalidad familiar(12), dificultades económicas y consumo de alcohol(13), así como la falta de apoyo social y para el descanso(11,14).

Los estudiantes de odontología requieren un mayor apoyo de su entorno, que los ayude a empoderarse de su carrera, disminuyendo así el agotamiento emocional que puedan experimentar al sentir frustraciones, desánimos, falta de compromiso, distanciamiento social y cognitivo, que en algunas ocasiones se percibe como una sobrecarga generada por las actividades propias de la disciplina escogida(14).

Dentro de las obligaciones de las entidades educativas, especialmente las universitarias, está el establecer mecanismos académicos, administrativos y de bienestar para promover adhesión y permanencia estudiantil a la vida universitaria, y que estos superen las dificultades que puedan encontrar en los programas académicos culminando así con éxito la carrera(3). Por lo anterior, si las universidades están interesadas en formar a un profesional de manera integral, que logre sus competencias académicas dentro de los tiempos estipulados, es decir, evitando la repetición de cursos y la deserción, las instituciones deberían considerar el entorno psicosocial de sus estudiantes para controlar, o disminuir factores de riesgo que comprometan su proyecto de vida académica.

Por tanto, el objetivo de este estudio fue determinar las relaciones entre las características sociodemográficas y familiares, con la funcionalidad familiar, el soporte de amigos y el rendimiento académico en los estudiantes de odontología de una universidad pública de la ciudad de Cartagena, Colombia; con el fin de ofrecer evidencia que permita identificar estudiantes con presencia de riesgo para su oportuno seguimiento y apoyo durante el transcurso de su carrera como profesional. 


\section{Materiales y métodos}

Estudio observacional de corte transversal que tuvo como población los estudiantes de odontología de una universidad pública de la región Caribe en Colombia. La muestra de 438 estudiantes fue seleccionada mediante un muestreo por convivencia, quienes debían estar matriculados al programa en el primer periodo del 2018 en cualquiera de los semestres, que aceptaran participar en el estudio y firmaran el consentimiento informado. Se excluyeron aquellos participantes que estuvieran recibiendo terapia familiar con un psicólogo, o en su defecto que estuvieran asistiendo a charlas con bienestar universitario con su familia.

El instrumento utilizado para la recolección de la información durante entrevistas realizadas a los estudiantes de manera personalizada y privada, previa prueba piloto realizada con otros estudiantes con características similares, fue de tipo encuesta con preguntas cerradas y de una sola respuesta, sobre variables sociodemográficas: edad (adolescentes, 1519 años y adulto Joven $\geq 20$ años), sexo (masculino, femenino), ingresos económicos (hasta 1 Salario Mínimo Mensual Legal Vigente (SMMLV) en Colombia, en 2018 y más de 1 SMMLV), Estrato socioeconómico (bajo-medio (estrato 1,2,3) y alto (4, 5), procedencia (urbana, rural), servicios de salud (público, privado), estado civil (soltero, casado). De igual forma, se evaluaron otros aspectos relacionados con el rol del estudiante al interior de su hogar (ser hijo, padre o madre), si el estudiante trabajaba, tenía hijos, si recibía o no el apoyo de sus padres. También, se indagó en el departamento académico de la institución universitaria, previo consentimiento de los estudiantes, por su promedio académico del último semestre cursado, según lo reportado en el reglamento estudiantil de la institución, categorizando esta variable en rendimiento académico bueno de $>3,5$ y rendimiento académico bajo $\leq 3,5$.

Con respecto a las características familiares y de la vivienda, se indagó por el tipo de unión de los padres (casados, solteros), quién trabajaba en la familia, años de estudios de los padres $(\leq 11$ años de estudio, $>11$ años), tenencia de vivienda (familiar o propia, arrendada), tipo de vivienda (casa, apartamento), presencia de hacinamiento (hasta 2 miembros por cuarto, $>2$ miembros), el número de miembros en la familia (hasta 4 miembros, $>4$ miembros), tipo de familia (familia nuclear, familia extensa, numerosa o ampliada, otros tipos de familia), tipo de riesgo familiar, según el tipo (tipo 1: Nuclear, tipo 2: No nuclear), ocupación de los padres (empleado, no empleado).

Se usó la escala Apgar familiar de Smilkteins (1978)(15), el cual es un instrumento validado y de autoinforme usado en muchos estudios para la valoración de la función familiar y el soporte de amigos(16). Se realizó una categorización de estas variables así: familia disfuncional (sí; no), falta de soporte en los amigos (sí; no). El Apgar es un instrumento que consta de cinco preguntas tipo Likert que se puntúan de 0 a 2 («casi siempre», 2 puntos; «a veces», 1 punto; «casi nunca», 0). Los resultados pueden variar entre 0 y 20 puntos; puntuaciones entre 18 y 20 indican funcionalidad familiar y las $<18$, disfuncionalidad familiar. Esta escala, que ha sido traducida y validada en castellano desde la validez de constructo y validez factorial, presentando una consistencia interna de 0,81 y fiabilidad pruebareprueba de $0,8127(17)$.

\section{Análisis de datos}

Los datos fueron analizados a partir de estadística descriptiva y análisis de relaciones bivariadas a partir de la prueba Chi cuadrado de Pearson, utilizando el programa estadístico STATA (Versión 14 para Windows). Se estimó la ocurrencia de la funcionalidad familiar y los factores asociados a la misma a través del cálculo de prevalencias.

\section{Consideraciones éticas}

Este estudio se realizó teniendo en cuenta las consideraciones éticas emitidas en la Resolución 8430 de 1993 del Ministerio de Salud de la República de Colombia(18). Igualmente, el estudio contó con aval del Comité de Ética en Investigaciones de la Universidad de Cartagena (Acta $\mathrm{N}^{\circ} 003$ ).

\section{Resultados}

\section{Características sociodemográficas de los estudiantes}

La muestra estuvo conformada por 438 estudiantes de odontología. Estos poseían una edad mínima de 15 años y máxima de 41 años, siendo el rango de 15 a 19 años el de mayor frecuencia (60\%). El 62,6\% pertenecía al sexo femenino y 81,9\% tenían una procedencia urbana y $96,3 \%$ afirmó ser soltero(a) (Tabla 1). 
Respecto al rol que asumía el estudiante, el 93,2\% afirmó asumir el rol de hijo(a), mientras que sólo el 6,8\% afirmó asumir el rol de padre, madre u otro. Asimismo, el 95,0\% manifestó no tener hijos; el $88,1 \%$ refirió trabajar mientras realizaba sus estudios y el 96,6\% recibía apoyo económico de sus padres (Tabla 1).

\section{Características sociodemográficas familiares}

El tipo de unión de los padres observado con mayor frecuencia fue casado o en unión libre $(77,2 \%)$. El $50,2 \%$ tenía ambos padres (madre y padre) y ambos trabajan en la familia, mientras que el $45,4 \%$ tenía sólo madre o padre, quien era el único que trabajaba en la familia. El ingreso económico de la familia más frecuente fue mayor a un SMMLV para Colombia. El $52,5 \%$ de los estudiantes pertenecían en a estratos socioeconómicos bajos $(1,2,3)$. Respecto a la escolaridad de los padres, tanto los padres como las madres poseían una educación equivalente a más de 11 años (media, técnica y superior) (Tabla 1).

\section{Características de la vivienda, composición y estructura familiar}

Los estudiantes manifestaron tener, en su mayoría, vivienda familiar o propia $(76,0 \%)$, siendo la casa el tipo de vivienda más frecuente (73,0\%). El 86,3\% afirmó que el número de miembros por cuartos de su vivienda se encontraba entre 1 a 2 , mientras que en un 53,2\% el número de miembros de la familia osciló entre 1 a 4 . Los tipos de familia más frecuentes fueron: nuclear $(46,1 \%)$, y extensa, numerosa o ampliada $(38,1 \%)$. El tipo familia según el riesgo mayormente observado fue el tipo 1 correspondiente a familia nuclear $(75,3 \%)$. Tanto los padres como las madres se encontraban empleados(as) en su mayoría, siendo la proporción de padres empleados mayor $(91,3 \%)$ en comparación a la proporción de madres empleadas $(65,7 \%)$ (Tabla 1$)$.

Tabla 1. Características sociodemográficas y familiares de los estudiantes de odontología, Cartagena

\begin{tabular}{lrc}
\hline \multicolumn{1}{c}{ Variable } & Número & (\%) \\
\hline Edad & & \\
Adolescentes (15-19 años) & 263 & 60,0 \\
Adulto joven ( $\geq 20$ años) & 175 & 40,0 \\
\hline Sexo & & \\
Femenino & 274 & 62,6 \\
Masculino & 164 & 37,4 \\
\hline Procedencia & & \\
Urbana & 359 & 81,9 \\
Rural & 79 & 18,1 \\
\hline Servicios
\end{tabular}

Servicios de salud

\begin{tabular}{|c|c|c|}
\hline Público & 135 & 30,8 \\
\hline Privado & 303 & 69,2 \\
\hline \multicolumn{3}{|l|}{ Estado civil } \\
\hline Soltero & 422 & 96,3 \\
\hline Casado & 16 & 3,7 \\
\hline \multicolumn{3}{|l|}{ Rol del estudiante } \\
\hline Hijo & 408 & 93,2 \\
\hline Padre, madre & 30 & 6,8 \\
\hline \multicolumn{3}{|l|}{ ¿El estudiante trabaja? } \\
\hline No & 386 & 88,1 \\
\hline Sí & 52 & 11,9 \\
\hline \multicolumn{3}{|l|}{ ¿El estudiante tiene hijos? } \\
\hline No & 416 & 95,0 \\
\hline Sí & 22 & 5,0 \\
\hline \multicolumn{3}{|c|}{ ¿El estudiante recibe apoyo de sus padres? } \\
\hline No & 15 & 3,4 \\
\hline Sí & 423 & 96,6 \\
\hline \multicolumn{3}{|l|}{ Tipo de unión de los padres } \\
\hline Casados & 338 & 77,2 \\
\hline Solteros & 100 & 22,8 \\
\hline \multicolumn{3}{|l|}{ ¿Quién trabaja en la familia? } \\
\hline Ambos padres & 220 & 50,2 \\
\hline Solo madre o padre & 199 & 45,4 \\
\hline Abuelos, tíos, otros & 19 & 4,4 \\
\hline \multicolumn{3}{|c|}{ Ingreso económico de la familia } \\
\hline Hasta 1 SMMLV & 43 & 9,8 \\
\hline Más de 1 SMMLV & 395 & 90,2 \\
\hline \multicolumn{3}{|l|}{ Estrato socioeconómico } \\
\hline Bajo $(1,2,3)$ & 230 & 52,5 \\
\hline Medio/alto (4 y 5) & 208 & 47,5 \\
\hline \multicolumn{3}{|l|}{ Escolaridad del padre } \\
\hline$\leq 11$ años de estudio & 162 & 37,0 \\
\hline$>11$ años & 276 & 63,0 \\
\hline \multicolumn{3}{|l|}{ Escolaridad de la madre } \\
\hline$\leq 11$ años de estudio & 180 & 41,1 \\
\hline$>11$ años & 258 & 58,9 \\
\hline \multicolumn{3}{|l|}{ Tenencia de vivienda } \\
\hline Familiar o propia & 333 & 76,0 \\
\hline Arrendada & 105 & 24,0 \\
\hline \multicolumn{3}{|l|}{ Tipo de vivienda } \\
\hline Casa & 320 & 73,0 \\
\hline Apartamento & 118 & 27,0 \\
\hline \multicolumn{3}{|l|}{ Hacinamiento } \\
\hline Hasta 2 miembros & 378 & 86,3 \\
\hline$>2$ miembros & 60 & 13,7 \\
\hline \multicolumn{3}{|c|}{ Número de miembros en la familia } \\
\hline Hasta 4 miembros & 233 & 53,2 \\
\hline$>4$ miembros & 205 & 46,8 \\
\hline \multicolumn{3}{|l|}{ Tipo de familia } \\
\hline Familia nuclear & 202 & 46,1 \\
\hline $\begin{array}{l}\text { Familia extensa, numerosa o } \\
\text { ampliada }\end{array}$ & 167 & 38,1 \\
\hline Otros tipos de familia & 69 & 15,8 \\
\hline \multicolumn{3}{|l|}{ Ocupación del padre } \\
\hline Empleado & 400 & 91,3 \\
\hline No empleado & 38 & 8,7 \\
\hline \multicolumn{3}{|l|}{ Ocupación de la madre } \\
\hline Empleada & 288 & 65,7 \\
\hline No empleada & 150 & 34,3 \\
\hline
\end{tabular}


Rendimiento académico, funcionalidad familiar y soporte de amigos

El $69,1 \%$ de los estudiantes poseía un rendimiento académico bueno (Figura 1A) y la mayoría se encontraban cursando semestres básicos $(63,7 \%)$ (Figura 1B). Respecto a la funcionalidad familiar, el
$55,2 \%$ de los estudiantes poseía una buena funcionalidad familiar, mientras que el 44,7\% poseía alguna disfunción familiar (Figura 1C). Por otro lado, el 81,7\% de los estudiantes poseía soporte de amigos, mientras que sólo el 18,2\% manifestó la ausencia de soporte de amigos (Figura 1D).
A

Rendimiento académico

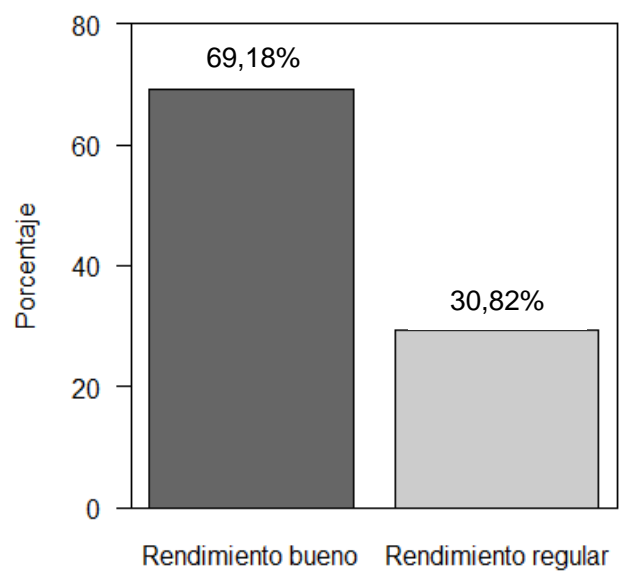

C

Funcionalidad familiar

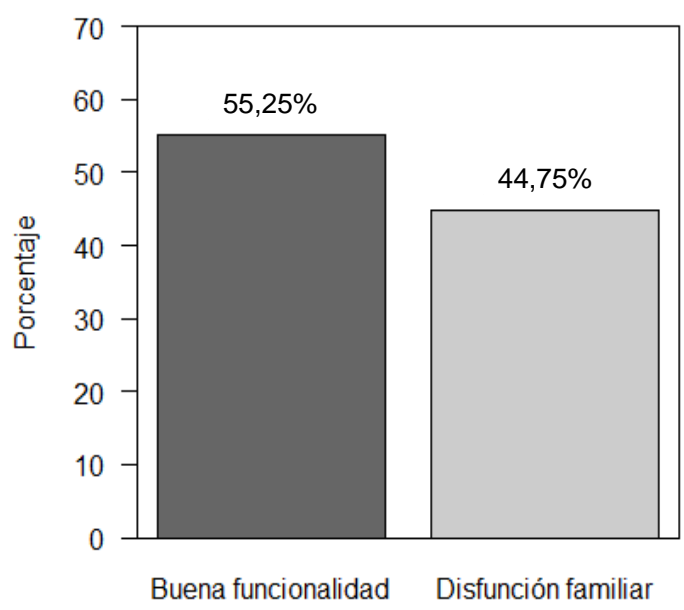

B

Semestre académico

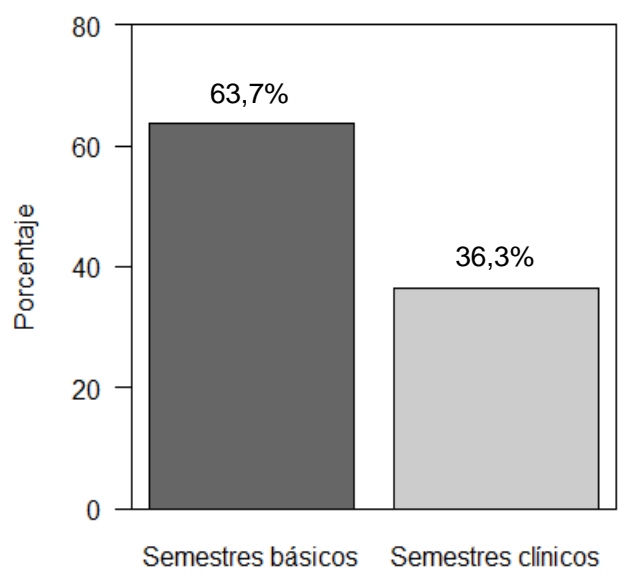

D

Soporte de amigos

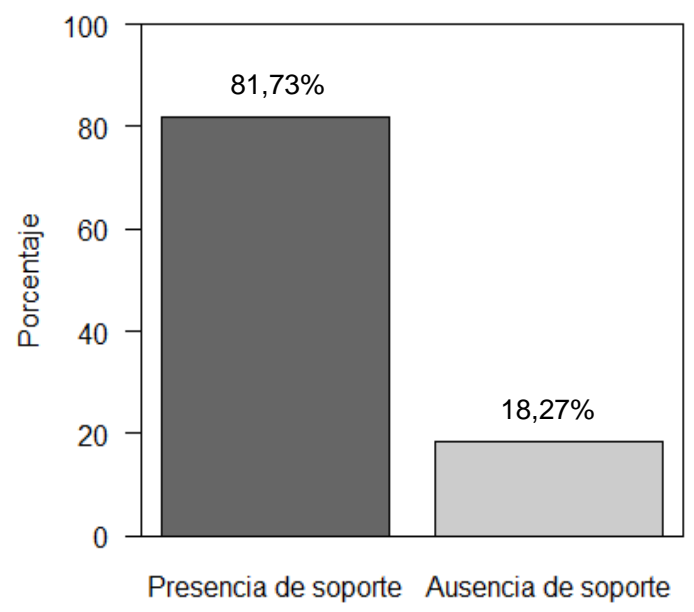

Figura 1. Descripción de las variables: A. Rendimiento académico, B. Semestre académico, C. Funcionalidad familiar y D. Soporte de amigos. 


\section{Análisis de las relaciones entre las variables de estudio}

$\mathrm{Al}$ examinar la relación entre la funcionalidad familiar, el soporte de amigos, el semestre en curso y el rendimiento académico (Figura 2), se encontró una relación estadísticamente significativa entre funcionalidad familiar y el tipo de semestre en curso (Figura 2B), observándose que la proporción de estudiantes con buena funcionalidad familiar fue mayor en aquellos que cursaban semestres básicos en comparación a los semestres clínicos $(p=0,0386)$.

A

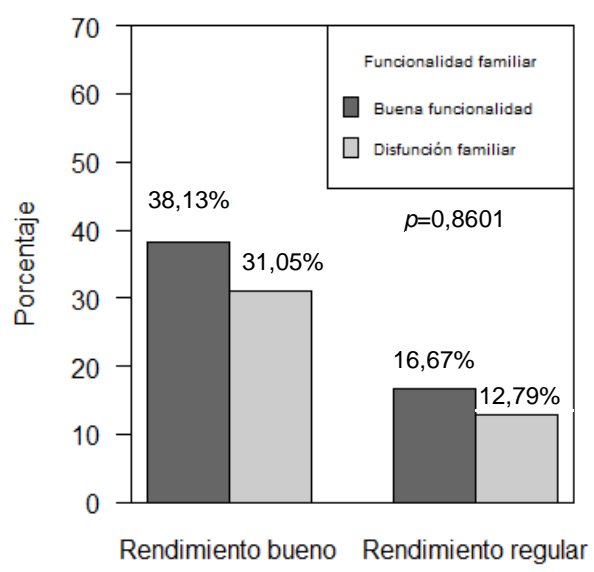

Rendimiento académico

C

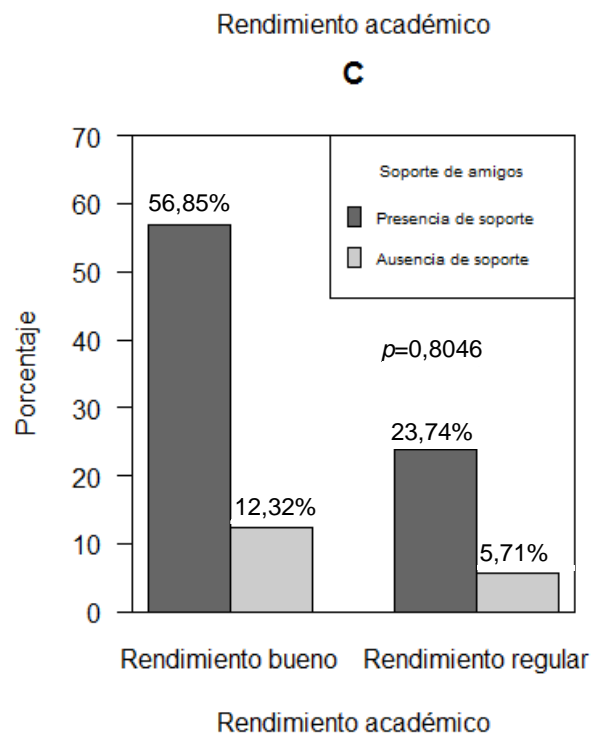

Respecto a la relación entre la funcionalidad familiar y las variables sociodemográficas del estudiante (Tabla 2), se observó una relación estadísticamente significativa entre la edad y la funcionalidad familiar, en la que la proporción de buena funcionalidad familiar fue mayor para aquellos con edades entre 15 a 19 años $(p=0,0029)$. Igualmente, al relacionar el soporte de amigos con las variables sociodemográficas, se obtuvo una relación estadísticamente significativa entre la procedencia y el soporte de amigos $(p=0,0230)$.
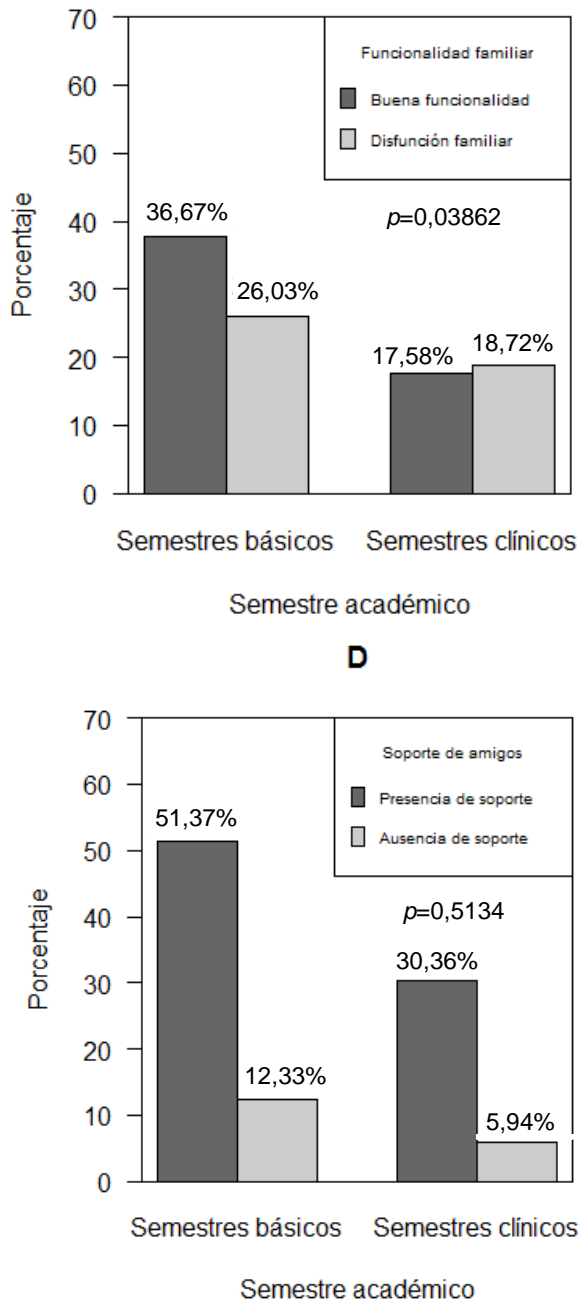

Figura 2. A. Relación entre la funcionalidad familiar y el rendimiento académico. B. Relación entre la funcionalidad familiar y el semestre académico. C. Relación entre el soporte de amigos y el rendimiento académico. D. Relación entre el soporte de amigos y el semestre académico. 
Tabla 2. Relación entre las variables funcionalidad familiar y soporte de amigos con las variables independientes

\begin{tabular}{|c|c|c|c|c|c|c|}
\hline \multirow[b]{2}{*}{ Variable } & \multicolumn{3}{|c|}{ Funcionalidad familiar } & \multicolumn{3}{|c|}{ Soporte de amigos } \\
\hline & $\begin{array}{c}\text { Buena } \\
\text { funcionalidad } \\
\text { n (\%) }\end{array}$ & $\begin{array}{c}\text { Disfunción } \\
\text { familiar } \\
\text { n (\%) }\end{array}$ & $P$ valor & $\begin{array}{c}\text { Presencia de } \\
\text { soporte } \\
\text { n (\%) }\end{array}$ & $\begin{array}{c}\text { Ausencia de } \\
\text { soporte } \\
\text { n (\%) }\end{array}$ & $P$ valor \\
\hline \multicolumn{7}{|l|}{ Edad } \\
\hline $15-19$ años & $161(36,7)$ & $102(23,3)$ & \multirow{2}{*}{$0,0029 *$} & $219(50,0)$ & $44(10,0)$ & \multirow{2}{*}{0,3719} \\
\hline $20-41$ años & $81(18,5)$ & $94(21,5)$ & & $139(31,7)$ & $36(8,3)$ & \\
\hline \multicolumn{7}{|l|}{ Sexo } \\
\hline Femenino & $151(34,5)$ & $123(28,5)$ & \multirow{2}{*}{1,0000} & $227(51,8)$ & $47(10,7)$ & \multirow{2}{*}{0,5154} \\
\hline Masculino & $91(20,8)$ & $73(16,2)$ & & $131(29,9)$ & $33(7,6)$ & \\
\hline \multicolumn{7}{|l|}{ Procedencia } \\
\hline Urbana & $202(46,2)$ & $157(35,8)$ & \multirow{2}{*}{0,4314} & $301(68,7)$ & $58(13,3)$ & \multirow{2}{*}{$0,0230^{*}$} \\
\hline Rural & $40(9,1)$ & $39(8,9)$ & & $57(13,0)$ & $22(5,0)$ & \\
\hline \multicolumn{7}{|l|}{ Rol del estudiante } \\
\hline Hijo & $230(52,5)$ & $178(40,6)$ & \multirow{2}{*}{0,1210} & $333(76,0)$ & $75(17,1)$ & \multirow{2}{*}{1,0000} \\
\hline Padre, madre, otro & $12(2,7)$ & $18(4,2)$ & & $25(5,7)$ & $5(1,2)$ & \\
\hline \multicolumn{7}{|l|}{ Seguridad social } \\
\hline Subsidiado & $66(15,1)$ & $69(15,7)$ & \multirow{2}{*}{0,0923} & $106(24,2)$ & $29(6,6)$ & \multirow{2}{*}{0,3034} \\
\hline Contributivo & $176(40,2)$ & $127(29,00)$ & & $252(57,5)$ & $51(11,7)$ & \\
\hline \multicolumn{7}{|l|}{ ¿El estudiante trabaja? } \\
\hline No & $218(49,7)$ & $168(38,4)$ & 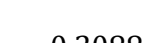 & $317(72,4)$ & $69(15,7)$ & \\
\hline Sí & $24(5,5)$ & $28(6,4)$ & 0,2088 & $41(9,4)$ & $11(2,5)$ & 0,7016 \\
\hline Estado civil & & & & & & \\
\hline Soltero, separado & $232(53,0)$ & $190(43,4)$ & 07354 & $344(78,5)$ & $78(17,8)$ & 07007 \\
\hline Casado, unión libre & $10(2,3)$ & $6(1,3)$ & 0,7354 & $14(3,2)$ & $2(0,5)$ & 0,7807 \\
\hline ¿El estudiante tiene hijos? & & & & & & \\
\hline No & $232(53,0)$ & $184(42,0)$ & & $341(77,8)$ & $75(17,1)$ & \\
\hline Sí & $10(2,3)$ & $12(2,7)$ & 0,4665 & $17(3,9)$ & $5(1,2)$ & 0,785 \\
\hline ¿El estudiante recibe apoyo $\mathrm{d}$ & dres? & & & & & \\
\hline No & $5(1,1)$ & $10(2,3)$ & 0,1408 & $14(3,2)$ & $1(0,2)$ & \\
\hline Sí & $237(54,1)$ & $186(42,5)$ & & $344(78,5)$ & $79(18,1)$ & 0,3992 \\
\hline Tipo de unión de los padres & & & & & & \\
\hline Casados, unión libre & $188(42,1)$ & $150(34,2)$ & & $279(63,7)$ & $59(13,5)$ & \\
\hline Separados, divorciados, viudos & $54(12,3)$ & $46(11,4)$ & 0,8635 & $79(18,0)$ & $21(4,8)$ & 0,5102 \\
\hline ¿Quién trabaja en la familia? & & & & & & \\
\hline Ambos padres & $124(28,3)$ & $96(21,9)$ & & $182(41,5)$ & $38(8,7)$ & \\
\hline Solo la madre o solo el padre & $110(25,1)$ & $89(20,3)$ & 0,4872 & $163(37,2)$ & $36(8,2)$ & 0,3004 \\
\hline Abuelos, tíos, otros & $8(1,8)$ & $11(2,6)$ & & $13(3,0)$ & $6(1,4)$ & \\
\hline Ingreso económico de la fami & & & & & & \\
\hline Hasta 1 SMMLV & $24(5,5)$ & $19(4,4)$ & & $35(8,0)$ & $8(1,8)$ & \\
\hline Más de 1 SMMLV & $218(49,8)$ & $177(40,3)$ & 1,000 & $323(73,7)$ & $72(16,5)$ & 1,0000 \\
\hline Estrato socioeconómico & & & & & & \\
\hline Bajo & $117(26,7)$ & $113(25,8)$ & $00 x=0$ & $183(41,8)$ & $47(10,7)$ & \\
\hline Medio/alto & $125(28,5)$ & $83(19,0)$ & 0,0653 & $175(40,0)$ & $33(7,5)$ & 0,2661 \\
\hline Escolaridad del padre & & & & & & \\
\hline Hasta 11 años & $82(18,7)$ & $80(18,3)$ & & $128(29,2)$ & $34(7,8)$ & \\
\hline$>11$ años & $160(36,5)$ & $116(26,5)$ & 0,1631 & $230(52,5)$ & $46(10,5)$ & 0,3164 \\
\hline Escolaridad de la madre & & & & & & \\
\hline Hasta 11 años & $83(19,0)$ & $97(22,1)$ & & $141(32,2)$ & $39(9,0)$ & \\
\hline$>11$ años & $159(36,3)$ & $99(22,6)$ & 0,0018* & $217(49,5)$ & $41(9,3)$ & 0,1575 \\
\hline Tenencia de vivienda & & & & & & \\
\hline Familiar, propia & $188(43,0)$ & $145(33,1)$ & 0 & $279(63,7)$ & $54(12,3)$ & $00<7$ \\
\hline Arrendada & $54(12,3)$ & $51(11,6)$ & 0,429 & $79(18,1)$ & $26(5,9)$ & $0,06 / 1$ \\
\hline Tipo de vivienda & & & & & & \\
\hline Casa & $179(40,9)$ & $141(32,2)$ & & $267(61,0)$ & $53(12,1)$ & 70 \\
\hline Apartamento & $63(14,4)$ & $55(12,5)$ & 0,7133 & $91(20,8)$ & $27(6,1)$ & 0,1679 \\
\hline
\end{tabular}




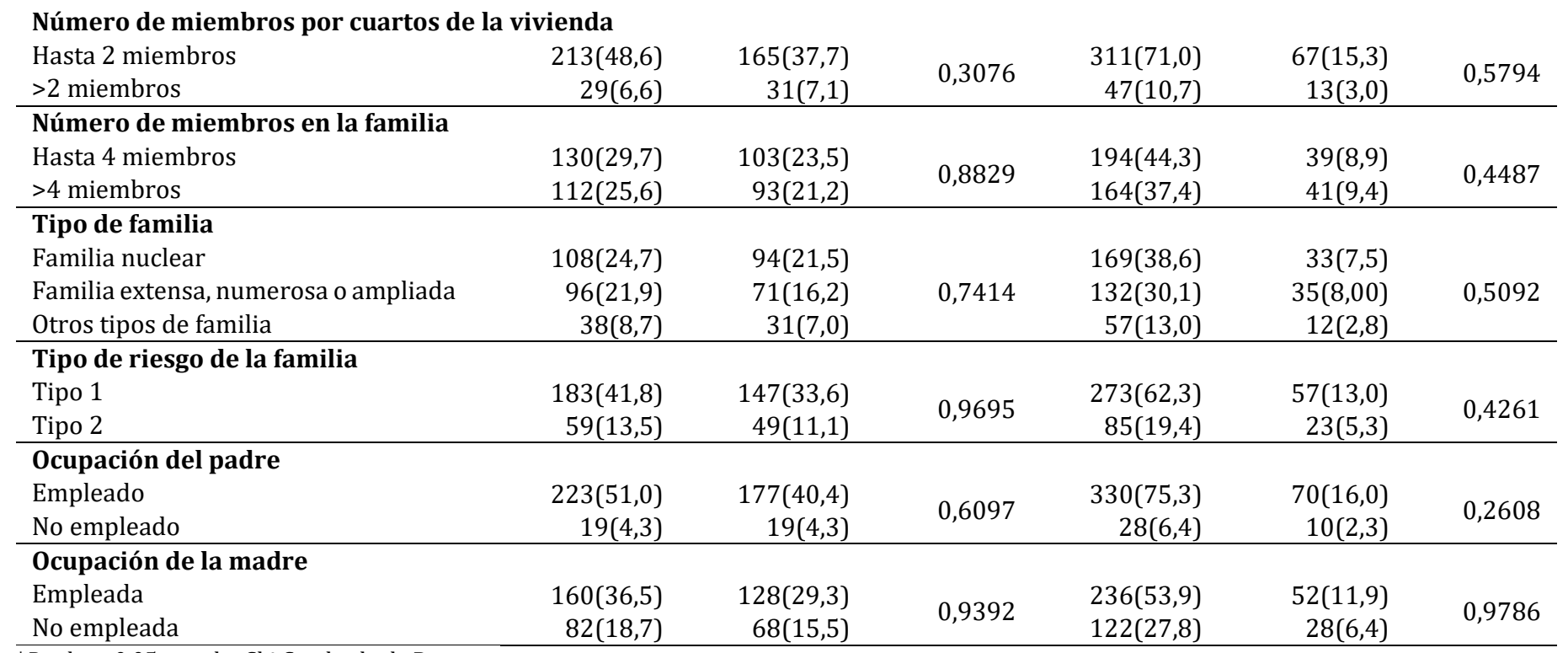

${ }^{*} P$ valor $<0,05$, prueba Chi Cuadrado de Pearson.

Sumado a lo anterior, se encontró una relación estadísticamente significativa entre la escolaridad de la madre y la funcionalidad familiar $(p=0,0018)$, observándose que aquellos estudiantes cuyas madres tenían una escolaridad mayor a 11 años de estudios, tuvieron una mayor proporción de buena funcionalidad familiar (Tabla 2). Finalmente, no se observó ninguna relación estadísticamente significativa con las variables relacionadas con la vivienda, la composición y estructura familiar (Tabla 2).

\section{Discusión}

Algunas características sociodemográficas del estudiante relacionadas con la funcionalidad familiar y el soporte de amigos, tales como, la edad del estudiante y su procedencia. Así mismo, características familiares como la escolaridad de la madre se asociaron con la funcionalidad familiar. También, el tipo de semestre (básico o clínicopráctico) en el que se encontraba el estudiante, mostró una relación con la funcionalidad familiar. Si bien hay estudios sobre el tema en estudiantes universitarios, estos abordan otros aspectos como las adicciones (internet, sustancias psicoactivas)(19), y son pocos los que estudian el entorno familiar y social, y cómo estos aspectos impactan en el rendimiento académico ${ }^{(20,21)}$.

En este estudio se encontró que los estudiantes de odontología perciben una buena funcionalidad familiar cuando están cursando los semestres básicos en comparación a los semestres clínicos. Al inicio de las carreras, los estudiantes están culminando la etapa de adolescencia y pueden contar mayor seguimiento de sus padres y percibir una mayor participación de su familia en sus actividades académicas ${ }^{(22)}$. A medida que van avanzando en la carrera, en donde se adquieren conocimientos sobre salud familiar, empiezan a realizar juicios que le pueden hacer cambiar su percepción sobre la vida y su entorno familiar(23,24).

En los semestres clínicos, dependiendo de su rendimiento académico y de las competencias adquiridas, algunos son más autónomos en sus decisiones. Algunas familias aún acompañan en este proceso, pero en otros casos, pueden estar ausentes las figuras de diálogo y comunicación entre los estudiantes y sus familias, posiblemente asumiendo que sus hijos tienen todo bajo control y esto pueda ser percibido por algunos como falta de apoyo. En consecuencia, a pesar de que las familias sigan ofreciendo ayuda, los estudiantes pueden no sentirlo así, debido a que tienen que desarrollar competencias propias y de su formación profesional, por esto, las percepciones de apoyo familiar pueden verse sesgadas en semestres más avanzados ${ }^{(25,26)}$.

Estos hallazgos muestran coherencia con la relación significativa observada entre la edad del estudiante y la percepción de funcionalidad familiar, lo cual puede ir de la mano con los cambios sociales esperados a vivir por ellos, al avanzar de adolescentes a adultos jóvenes. Entre estos cambios se encuentra que los estudiantes prefieren pasar más tiempo con los amigos que con la familia, dejando a la familia en un 
segundo plano, lo cual pueda malinterpretarse como una ausencia de apoyo. Estos resultados coinciden con los reportados por otros estudios a nivel local con estudiantes de odontología pero en una escuela $\operatorname{privada}^{(27)}$.

León-Sanchez et al.(28), reportaron que existe una relación significativa entre la disfunción familiar y el nivel educacional de los padres, lo cual influye en la transmisión de las conductas de los padres a los hijos, y que estos puedan interiorizarlas y hacerlas propias. En este orden de ideas, contar con padres con un nivel de escolaridad superior a 11 años podría representar un mejor apoyo para los estudiantes de odontología como lo confirman los resultados del presente estudio. La sobrecarga del estudiante y estrés por los exámenes puede verse como una dificultad en aquellos alumnos cuya madre posee estudios medios o básicos, así como la falta de control sobre el propio rendimiento académico y la baja autoestima académica según lo reportado por Soto et al(29).

Algunos autores como Morales et al.(30), indican que, si los estudiantes están habituados a ver leer y utilizar libros, estarán más motivados al estudio y mucho más, si estos hábitos son vistos en casa desde sus propios padres. Otros autores manifiestan que la formación académica de los padres se relaciona positivamente con la ansiedad ante los exámenes de los estudiantes, lo cual demuestra que el apoyo de los padres es fundamental para generar motivación, concentración, esfuerzo y una buena actitud(31,32).

Todo lo anterior denota la importancia que tiene no sólo el apoyo familiar percibido por los estudiantes de odontología, sino el apoyo social recibido por parte de sus amigos. Algunos estudiantes reportaron presentar un buen soporte de amigos cuando logran un buen rendimiento académico, probablemente por el desarrollo de actividades realizadas en grupo que dan seguridad a la hora de realizar sus trabajos académicos y apoyan desde el proceso de hetero aprendizaje(33). Jiménez et al., reportaron que el trabajo en grupo genera en los estudiantes compromiso en las tareas asignadas, lo que a su vez les permite incorporar mayores habilidades sociales y aumentar su aprendizaje ${ }^{(34)}$.

En los semestres básicos se desarrollan más actividades grupales mientras que en los semestres clínicos, predominan los trabajos individuales, lo cual puede conllevar a que el estudiante perciba que se encuentra solo, al tener que enfrentar el paso de la teoría a la práctica y tener que desarrollar destrezas, competencias del saber hacer $y$ habilidades comunicativas al atender pacientes de manera individual. Estas son circunstancias que le pueden generar estrés al estudiante, por ser situaciones nuevas e impactar sobre su rendimiento académico.

Otros estudios han reportado que estudiantes con problemas de depresión, ansiedad y estrés perciben un bajo apoyo social(35). Por lo tanto, se resalta la importancia del soporte de amigos en el proceso de aprendizaje durante el desarrollo de sus carreras, al representar este soporte una red de apoyo con la cual pueden compartir sus dificultades y emociones, además de convertirse en un buen recurso de afrontamiento. Estos son aspectos para considerar por parte de las facultades y escuelas de odontología, para que se puedan generar nuevas estrategias de aprendizaje desde la implementación de trabajos más colaborativos entre pares, que permitan el desarrollo conjunto de habilidades(36).

El soporte de amigos estuvo relacionado significativamente con la procedencia de los estudiantes. Para muchos estudiantes no es fácil el dejar de vivir en zonas rurales, en donde el estilo de vida es mucho más calmado y tranquilo. Dejar su casa, su familia y llegar a una nueva ciudad con costumbres y realidades diferentes, puede generar retos de adaptación y la construcción de nuevas amistades y redes de apoyo(14,37). Por su parte, una gran proporción considerable de los estudiantes incluidos en el presente estudio procedían de áreas rurales, quienes deben realizar mayores esfuerzos económicos para solventar sus estudios. Estos factores pueden incidir en el bienestar personal y familiar del individuo(38), lo cual debería ser de consideración para las instituciones de educación superior(39).

Los hallazgos del presente estudio deben ser interpretados o analizados a la luz de las siguientes limitaciones: se trata de un estudio de corte transversal que no permite extrapolar las conclusiones a otras poblaciones y el potencial sesgo de información correspondiente al usar el instrumento Apgar familiar, ya que al conocer qué se está midiendo se pueden lograr respuestas socialmente esperadas. No obstante, dentro de sus fortalezas, se encuentra el realizar investigaciones con estudiantes de odontología, en quienes la observación de su estado de salud familiar resulta de vital importancia, dado que estos se convertirán en un 
futuro, en los cuidadores profesionales de la salud de las comunidades.

Sería conveniente considerar el desarrollo de estudios cualitativos que indaguen más a fondo sobre las percepciones de la funcionalidad familiar de los universitarios, que ofrezcan más claridad frente a las dificultades vividas por ellos en su entorno, y que permitan mejorar las preguntas relacionadas con la funcionalidad en estudios cuantitativos.

Los aportes de este estudio dan luces a las instituciones para considerar al estudiante como un individuo que, a pesar de contar muchas veces con una mayoría de edad, son jóvenes que recurren a su familia y amigos como mecanismo de apoyo. Para lo cual sería importante involucrar estos núcleos socializadores en las intervenciones académicas y de Bienestar Universitario, con el ánimo de lograr un mejor desarrollo académico y psicosocial durante el trascurso de su carrera.

\section{Conclusiones}

Existen factores tanto sociodemográficos como familiares que se relacionan con la funcionalidad familiar y el soporte de amigos en estudiantes de odontología. La identificación de estos factores podría permitir determinar qué aspectos deben ser tomados en cuenta para el diseño e implementación de intervenciones académicas, las cuales puedan brindarles mayor apoyo, para que estos se desarrollen académica y psicosocialmente de manera óptima durante sus estudios universitarios.

Conflicto de intereses: Ninguno declarado por los autores.

\section{Referencias}

1. Sistema Nacional de Información de la educación superior. Lista de Informes departamentales de Educación Superior. 2016 [consultado 22 Noviembre 2020]. Disponible en: https://www.mineducacion.gov.co/sistemasdeinformacion /1735/w3-article-212352.html

2. Gómez Pacheco JP, Montero Campo DI. Calidad de vida en relación con la salud física y mental en estudiantes de la Universidad de la Costa [Tesis de grado]. Barranquilla, Colombia: Universidad de la Costa; 2019. Disponible en: https://repositorio.cuc.edu.co/handle/11323/4827

3. López Mero P, Barreto Pico A, Mendoza Rodríguez ER, del Salto Bello MWA. Bajo rendimiento académico en estudiantes y disfuncionalidad familiar. Medisan. 2015 [consultado 22 Noviembre 2020];19(9):1163-6. Disponible en: $\quad$ http://scielo.sld.cu/scielo.php?pid=S102930192015000900014\&script=sci_arttext\&tlng=en
4. de Oliveira ES, Rodrigues da Silva AF, Barbosa da Silva KC, Carneiro Moura TV, de Araújo AL, Vilarouca da Silva AR. Stress and health risk behaviors among university students. Rev Bras Enferm. 2020;73(1):e20180035. DOI: 10.1590/0034-7167-2018-0035.

5. Sandoval Barrientos S, Dorner París A, Véliz Burgos A. Bienestar psicológico en estudiantes de carreras de la salud. Investig en Educ Médica. 2017;6(24):260-6. DOI: 10.1016/j.riem.2017.01.004.

6. Pérez Díaz F, Cartes-Velásquez R. Estrés y burnout en estudiantes de Odontología: una situación preocupante en la formación profesional. Edumecentro. 2015;7(2):179-90. Disponible http://scielo.sld.cu/scielo.php?script=sci_arttext\&pid=S207 7-28742015000200014\&lng=es

7. Torales J, Barrios I, Samudio A, Samudio M. Self-perceived social support in medical students of the National University of Asunción, Paraguay. Educ Medica. 2018;19(3):313-7. DOI: 10.1016/j.edumed.2017.07.001.

8. Schmitter M, Liedl M, Beck J, Rammelsberg P. Chronic stress in medical and dental education. Med Teach. 2008;30(1):979. DOI: 10.1080/01421590701769571.

9. Basudan S, Binanzan N, Alhassan A. Depression, anxiety and stress in dental students. Int J Med Educ. 2017;8:179-86. DOI: 10.5116/ijme.5910.b961.

10. Sáenz Duran S, González Martínez F, Díaz Cárdenas S. Hábitos y Trastornos Alimenticios asociados a factores Sociodemográficos, Físicos y Conductuales en Universitarios de Cartagena, Colombia. Rev Clínica Med Fam. 2011;4(3):193204. DOI: $10.4321 / \mathrm{s} 1699-695 \times 2011000300003$.

11. Díaz Cárdenas S, Martínez Redondo M, Zapata Teherán AM. Academic performance and quality of life related to health in dentistry students. Salud Uninorte. 2017;33(2):139-51. DOI: 10.14482/sun.33.2.10540.

12. Díaz Cárdenas S, Arrieta Vergara K, González Martinez F. Estrés académico y funcionalidad familiar en estudiantes de Odontología. Rev Clínica Med Fam. 2012;5(3):217-8. DOI: 10.4321/s1699-695x2012000300014.

13. Arrieta Vergara KM, Díaz Cárdenas S, González Martínez F. Síntomas de depresión y ansiedad en jóvenes universitarios: prevalencia y factores relacionados. Rev Clínica Med Fam. 2014;7(1):14-22. DOI: 10.4321/s1699$695 \times 2014000100003$.

14. Arrieta Vergara K, Díaz Cárdenas S, González Martínez F. Síntomas de depresión, ansiedad y estrés en estudiantes de odontología: prevalencia y factores relacionados. Rev Colomb Psiquiatr. 2013;42(2):173-81. DOI: 10.1016/S00347450(13)70004-0.

15. Suarez Cuba MA, Alcalá Espinoza M. Apgar Familiar: Una Herramienta Para Detectar Disfunción Familiar. Rev Médica La Paz. 2014 [consultado 22 Noviembre 2020];20(1):53-7. http://www.scielo.org.bo/scielo.php?pid=S172689582014000100010\&script=sci_arttext

16. Rangel JL, Valerio L, Patiño J, García M. Funcionalidad familiar en la adolescente embarazada. Rev la Fac Med. 2004;47(1):24-7. Disponible

https://www.medigraphic.com/cgibin/new/resumen.cgi?IDARTICULO $=646$

17. Bellón Saameño JA, Delgado Sánchez A, Luna del Castillo JD, Lardelli Claret P. Validity and reliability of the Duke-UNC-11 questionnaire of functional social support. Aten Primaria. 1996;18(4):153-63. Disopnible

en: https://www.elsevier.es/es-revista-atencion-primaria-27articulo-validez-fiabilidad-del-cuestionario-apoyo-14325 
18. Ministerio de Salud y Protección Social. Resolución 8430 de 1993 por la cual se establecen las normas científicas, técnicas y administrativas para la investigación en salud. 1993. Disponible en: https://www.minsalud.gov.co/sites/rid/Lists/BibliotecaDi gital/RIDE/DE/DIJ/RESOLUCION-8430-DE-1993.PDF

19. Castaño Castrillón JJ, Páez Cala ML. Funcionalidad familiar y tendencias adictivas a internet y a sustancias psicoactivas en estudiantes universitarios. Psicol desde el Caribe. 2019;36(2):177-206. Disponible en: https://www.redalyc.org/journal/213/21363396003/

20. Munares-García O, Zagaceta-Guevara Z, Solís Rojas M. Función familiar y rendimiento académico en estudiantes de obstetricia de una universidad pública de Perú. Matronas prof. 2017;18(1):e1-e9. Disponible en: https://matronasprofesion.es/archivo/vol-18-num-12017/?ver=2-1-5

21. Díaz Cárdenas S, Arrieta Vergara K, González Martínez F. Academic stress and family function in dentistry students. Salud Uninorte. 2014;30(2):121-32. Disponible en: http://www.scielo.org.co/pdf/sun/v30n2/v30n2a04.pdf

22. Srivastava R, Jyoti B, Pradhan D, Kumar M, Priyadarshi P. Evaluating the stress and its association with stressors among the dental undergraduate students of Kanpur city, India: A cross-sectional study. J Educ Health Promot. 2020;9:56. Disponible

en: https://www.ncbi.nlm.nih.gov/pmc/articles/PMC7255575

23. Rodríguez Rodríguez D, Guzmán Rosquete R. Rendimiento académico: Y factores sociofamiliares de riesgo Variables personales que moderan su influencia. Perfiles Educ. 2019;41(164):118-34.

DOI: 10.22201/iisue.24486167e.2019.164.58925.

24. Cudris Torres L, Borja Acuña Y, Bahamon MJ, Morón Cotes ML. Capitulo 9: Análisis de la comunicación familiar: Un estudio realizado con estudiantes universitarios de Valledupar. Percepción de la comunicación con padres y rendimiento académico en estudiantes universitarios de Valledupar. Estudios actuales en Psicología; 2016. Disponible en:

https://bonga.unisimon.edu.co/bitstream/handle/20.500.1 2442/1120/Cap_9_Análisis_Comunicación.pdf?sequence=1 4\&isAllowed $=\mathrm{y}$

25. Abu-Ghazaleh SB, Sonbol HN, Rajab LD. A longitudinal study of psychological stress among undergraduate dental students at the University of Jordan. BMC Med Educ. 2016;16(1):90. DOI: 10.1186/s12909-016-0612-6.

26. Gabalán Coello J, Vásquez Rizo F-E. Rendimiento académico universitario y asistencia a clases: Una visión. Rev Educ. 2017;41(2). DOI: 10.15517/revedu.v41i2.18477.

27. Madera Anaya MV, Fétis Navarrete GR, Tirado Amador LR. Empatía Y Factores Relacionados En Estudiantes De Odontología De La Universidad De Cartagena. Rev Colomb Investig en Odontol. 2014;5(14):69. doi: $10.25063 / 21457735.176$.

28. León Sánchez D, Camacho Delgado R, Valencia Ortíz M del R, Rodríguez-Orozco AR. Percepción de la función de sus familias por adolescentes de la enseñanza media superior. Rev Cubana Pediatr. 2008;80(3). http://scielo.sld.cu/scielo.php?script=sci_arttext\&pid=S003 4-75312008000300004

29. Da Cuña-Carrera I, Soto-González M, Gutiérrez-Nieto M, Barreira-Salgado A. Nivel educativo de los progenitores como factor mediador del estrés académico. Rev la Fund Educ
Médica.

2018;21(1):23-9.

DOI:

10.33588/fem.211.929\#: :text=10.33588/fem.211.929.

30. Morales P. Investigación e innovación educativa. REICE Rev Iberoam sobre Calidad Efic Cambio Educ. 2016;8(2):47-73. Disponible en: https://revistas.uam.es/reice/article/view/5360

31. Nagpal M, Sinha C. Perceived parental pressure and academic achievement among students: Exploring the mediating effect of test anxiety among school students. J Educ Psychol. 2016. Disponible en: https://ojep.org/wpcontent/uploads/2020/05/2016NS.pdf

32. López-Angulo Y, Pérez-Villalobos MV, Cobo-Rendón RC, DíazMujica AE. Social support, gender and knowledge area over self-perceived academic performance in Chilean university students. Form Univ. 2020;13(3):11-8. DOI: 10.4067/S071850062020000300011.

33. Gökçearslan Ş, Uluyol Ç, Şahin S. Smartphone addiction, cyberloafing, stress and social support among university students: A path analysis. Child Youth Serv Rev. 2018;91:4754. DOI: 10.1016/j.childyouth.2018.05.036.

34. Ángel Jiménez G, Pareja Cadavid AM, Puerta Lasprilla KV. El trabajo en equipo como modalidad de enseñanzaaprendizaje. Rev iberoam Educ investi Enferm. 2013;3(1):9$15 . \quad$ Disponible en: https://www.enfermeria21.com/revistas/aladefe/articulo/ 58/el-trabajo-en-equipo-como-modalidad-de-ensenanzaaprendizaje/

35. Álvarez Rúa LJ, Carmona Rendón YA, Holguín Vásquez VA, Ortiz Parra MA, Patiño Arango SE, Vélez Peláez S, et al. Salud mental en estudiantes de odontología de una Universidad Pública de Medellín (Colombia) y sus factores relacionados. Rev Nac Odontol. 2019;15(29):1-19. DOI: 10.16925/23574607.2019.02.08.

36. Caballero M del C. Estilos de aprendizaje en estudiantes de la carrera de odontología de la Universidad Privada Abierta Latinoamericana - UPAL. Ad Astra. 2015;6(4):111-6. Disponible en: http://www.revistasbolivianas.org.bo/pdf/rcma/v6n4/v6n 4_a02.pdf

37. Fernández-Lasarte O, Ramos-Díaz E, Axpe Sáez I. Rendimiento académico, apoyo social percibido e inteligencia emocional en la universidad. Eur J Investig Heal Psychol Educ. 2019;9(1):39-49. DOI: 10.30552/ejihpe.v9i1.315.

38. Chacón-Cuberos R, Martínez-Martínez A, Puertas-Molero P, Viciana-Garófano V, González-Valero G, Zurita-Ortega F. Bienestar social en la etapa universitaria según factores sociodemográficos en estudiantes de educación. Rev Electrónica Investig Educ. 2020;22:e03. DOI: 10.24320/redie.2020.22.e03.2184.

39. Luna D, García-Reyes S, Soria-González EA, Avila-Rojas M, Ramírez-Mollina V, García-Hernández B, et al. Estrés académico en estudiantes de odontología: asociación con apoyo social, pensamiento positivo y bienestar psicológico. Investig en Educ Médica. 2020;9(35):8-17. DOI: 10.22201/facmed.20075057e.2020.35.2020. 